\title{
Competencias emocionales básicas para profesores en formación
}

\author{
Basic emotional competencies for teacher trainees \\ Competências emocionais básicas para professores estagiários
}

ARTíCULO DE INVESTIGACIÓN

\author{
Yuraima Yannine de los Ángeles Zambrano-Mendoza \\ yuraima.zambrano@uasb.edu.bo \\ https://orcid.org/0000-0002-7732-5685 \\ Universidad Andina Bolívar, Sucre-Bolivia
}

Recibido 11 de mayo 2021 | Arbitrado y aceptado 15 de junio 2021 | Publicado en 01 julio 2021

\section{RESUMEN}

El articulo tiene tiene como finalidad determinar las competencias emocionales básicas y la relación con el proceso de enseñanza y aprendizaje de los profesores en formación en Educación Básica de la Universidad Nacional de Loja. Bajo un enfoque mixto, se aplicaron diferentes instrumentos con base en los objetivos planteados. Se realizó una integración de ambos enfoques, para ampliar la perspectiva de respuestas de docentes y de los adultos. Mediante los resultados obtenidos se identificaron elementos facilitadores y obstaculizadores del quehacer docente sobre las emociones de sus estudiantes, se logró constatar una deficiencia en el profesional en educación en cuanto al desarrollo conocimiento de la temática, la falta de estrategias aplicables a las emociones de sus estudiantes, la poca aplicación de habilidades como la empatía y la escucha. Se concluye la pertinencia de brindar al personal docente un modelo de aprendizaje que comprende la motivación, las estrategias de aprendizaje e inteligencia emocional

Palabras clave: Educación emocional; Maestros, Adultos; Competencias emocionales, Desarrollo emocional
ABSTRACT

The purpose of this article is to determine the basic emotional competencies and their relationship with the teaching and learning process of teachers in training in Basic Education at the National University of Loja. Under a mixed approach, different instruments were applied based on the proposed objectives. Both approaches were integrated in order to broaden the perspective of teachers' and adults' responses. Through the results obtained, facilitating and hindering elements of the teachers' work on the emotions of their students were identified, and a deficiency was found in the education professional regarding the development of knowledge of the subject, the lack of strategies applicable to the emotions of their students, and the little application of skills such as empathy and listening. We conclude the relevance of providing teachers with a learning model that includes motivation, learning strategies and emotional intelligence.

Key words: Emotional education; Teachers, Adults; Emotional competencies, Emotional development

\section{RESUMO}

O objetivo deste artigo é determinar as competências emocionais básicas e sua relação com o processo de ensino e aprendizagem dos professores estagiários na Educação Básica da Universidade Nacional de Loja. Sob uma abordagem mista, diferentes instrumentos foram aplicados com base nos objetivos declarados. Foi realizada uma integração de ambas as abordagens, a fim de ampliar a perspectiva das respostas dos professores e dos adultos. Através dos resultados obtidos, foram identificados elementos facilitadores e dificultadores no trabalho de ensino sobre as emoções de seus alunos, uma deficiência do profissional na educação em termos do desenvolvimento do conhecimento do assunto, a falta de estratégias aplicáveis às emoções de seus alunos, a pouca aplicação de habilidades como a empatia e a escuta. Concluímos a relevância de proporcionar aos professores um modelo de aprendizagem que inclua motivação, estratégias de aprendizagem e inteligência emocional.

Palavras-chave: Educação emocional; Professores; Adultos; Competências emocionais; Desenvolvimento emocional 


\section{INTRODUCCIÓN}

Las competencias emocionales son el conjunto de conocimientos, capacidades, habilidades y actitudes que permiten a las personas tomar conciencia, comprender, expresar y regular las emociones de forma apropiada en las diferentes circunstancias de la vida, para que los estudiantes las desarrollen y disfruten del bienestar personal y social

Así, en el ámbito educativo, particularmente en el campo universitario existen cambios importantes que buscan optimizar los procesos de enseñanza y aprendizaje, para ejercer actividades escolares con la calidad, aplicando diferentes enfoques como es el aprendizaje basado en competencias que orienten una formación integral. Al respecto, Pegalajar y López, (2015) sostienen que la importancia de la formación por competencias emocionales, se basa en fortalecer el proceso educativo, sensibilizar al docente en formación, con ello se tome conciencia de sí mismo al manejar y reconocer las emociones y motivaciones con el entorno.

Siguiendo la línea de pensamiento, las instituciones de educación superior asumen el reto de contribuir en la formación de ciudadanos, responsables, críticos y comprometidos con el desarrollo de la sociedad, lo cual permite desenvolverse en la vida, para ello es fundamental afianzar el proceso de aprendizaje, también en el ámbito emocional.

El modelo educativo por competencias, en estrecha relación con el paradigma socio constructivista, persigue una convergencia entre los campos social, afectivo, las habilidades cognoscitivas, psicológicas, sensoriales, motoras, del individuo, en donde el aprendizaje potencia una integración de las disciplinas del conocimiento, las habilidades genéricas y la comunicación de ideas (Argudin, 2005).

Por tanto, cobra protagonismo en este escenario la formación en competencias emocionales secundarias, como una urgencia al cambio metodológico, con énfasis en la formación práctica de los estudiantes, articulando el contacto con situaciones reales de aprendizaje y contribuyan al desarrollo de habilidades para la adaptación a los cambios anímicos en el contexto educativo y las relaciones interpersonales.

A partir de la revisión de la literatura existen diferentes tipos de competencias, entre las que destacan las competencias cognitivas, las técnicas, las relacionales y las emocionales; la última como se planteó anteriormente es el desarrollo de conocimientos y habilidades sobre las emociones para capacitar al individuo y afrontar mejor los retos que se planten en la vida. Todo ello tiene como finalidad aumentar el bienestar personal y social.

Es indudable que la postura de varios investigadores se centra en el aporte de las competencias emocionales en la resolución de conflictos, más aún cuando se los vincula con la educación. Criterios válidos señalan las causas de aceptación y de rechazo en el aula, así como la participación del profesorado en la prevención de conflictos interpersonales, en donde es importante realizar una intervención educativa en la aplicación de programas emocionales y de mediación que facilite a los profesores tratar frecuentemente con los estudiantes la temática para sensibilizar sobre las relaciones interpersonales.

Se considera acertado señalar que la práctica educativa universitaria está orientada 
a la formación profunda de profesionales con habilidades, conocimientos, destrezas, aptitudes y actitudes que ayuden a desenvolverse de manera eficiente en la carrera profesional, por ello es prioritario emplear un modelo educativo basado en competencias emocionales básicas que garantice su adquisición, oriente que los docentes en formación consoliden los conocimientos teóricos, con habilidades emocionales que contribuya el equilibrio en la calidad de vida y centrar la práctica educativa en asistir las competencias emocionales interpersonales, mejorando el clima en el aula y garantizar el desarrollo integral de los estudiantes.

El desarrollo y evaluación de competencias emocionales con el paso del tiempo demanda el interés de varias disciplinas, en donde se enfatiza los programas de intervención, buscar la eficacia de la aplicación con estrategias vinculadas al bienestar personal, con ello el aporte en la enseñanza de los estudiantes. Torrijos, (2016) afirma que es innegable la importancia de las emociones en el terreno educativo. Cualquier acción educativa que se precie constituye un intercambio de emociones constante, tanto por el docente del discente, determinando la relación educativa, por consiguiente, la influencia en el proceso de enseñanza-aprendizaje.

A todo esto, es necesario analizar los efectos de los programas de educación emocional en los profesores, tal es el caso del estudio realizado por García, (2017) quién sostiene la existencia de mejora en competencias emocionales y el clima de centro educativo, después de implementarlo, concluyendo que hay evolución positiva en las competencias emocionales del profesorado entre el pretest y el postest. Se confirma la relación entre el desarrollo de competencias emocionales de los tutores y la satisfacción con la vida, comprobando la existencia de correlación significativa positiva entre las competencias emocionales y el engagement.

Otra investigación realizada por Bisquerra y Pérez, (2007) afirman que las aplicaciones de las competencias emocionales en la empresa, la salud y la educación establecen la difusión de una educación emocional y la contribución efectivamente en la educación formal como en la familia al fortalecer las relaciones interpersonales, con ello la disminución de la conflictividad en el rendimiento académico y en el bienestar.

Estudios en América del Sur como el de Sánchez, (2019) destaca "La docencia es un trabajo emocional", debido a que necesita del desarrollo de habilidades emocionales, por lo tanto, tiene que incluirse en la formación inicial del profesorado. Se destaca en la investigación la necesidad que la educación incluya el ámbito emocional en la práctica docente como aspecto básico del proceso educativo, la articulación del desarrollo de competencias emocionales como la conciencia y regulación emocional, autonomía emocional, competencia social, habilidades de vida y bienestar, manteniendo el dualismo cognición-emoción.

Saludable es acotar que uno de los aspectos que se deben considerar en la actualidad es el cambio radical en el proceso enseñanza-aprendizaje, ocasionado por la pandemia de COVID-19 que obligó el cierre de centros educativos y el nuevo modelo implica la educación virtual, el cual según la UNESCO, (2020) provoco tanto en estudiantes como docentes diferentes manifestaciones emocionales negativas como estrés, ansiedad y miedo, por lo que recomienda desarrollar habilidades de aprendizaje socioemocional, que permitirá a los principales 
actores a responder emocionalmente de una forma más equilibrada.

En esta misma línea, Cáceres, (2020) refiere que la educación post pandemia debe ajustarse a la nueva realidad y direccionarse a disminuir la segregación social que dejo en evidencia el COVID-19, para ello resulta necesario que el enfoque de la educación sea a nivel de aprendizaje, emocional y social. En concordancia, Camargo et al., (2020) Pires, Zen, Freitas, Arruda y Gemperli (2020) mencionan que la pandemia por COVID-19, por la duración se convierte en una de las situaciones epidémicas de mayor trascendencia, por tanto es importante la formación de personal capacitado que detecte las necesidades de los estudiantes, en un entorno virtual que contemple el aprendizaje emocional.

El éxito académico de los estudiantes en las instituciones de Educación Superior es el objetivo fundamental del proceso pedagógico. Sin embargo, esta aspiración implica enfrentar eficientemente, los desafíos, retos, complicaciones que se presenten en el desarrollo de la función docente, precisamente tomando como referencia el escenario de aprendizaje áulico, donde se tiene que ejecutar metodologías de trabajo que innoven el proceso de educativo y garantice que los estudiantes tengan acceso a educación de calidad.

Cabe mencionar que en los últimos años en Ecuador el proceso educativo viene dando pasos de mejora para garantizar el aprendizaje de los alumnos, se aplican mecanismos para capacitar a docentes en estrategias y herramientas pedagógicas, pero existen desudas pendientes en la capacitación en el campo emocional y su a importancia en el sistema educativo; instituciones educativas que presentan problemas de comportamiento $y$ rendimiento académico del alumnado, alto grado de irresponsabilidad, estrés, desanimo, problemas para relacionarse o no mantienen buenas relaciones sociales en estudiantes universitarios, por cuanto es de importancia proponer una intervención educativa desde el ámbito emocional.

Razón por la cual, uno de los problemas centrales se basa en el desconocimiento que se tiene, no solo del importante segmento de los formadores profesionales que requieren de estrategias alternativas para optimizar los procesos de aprendizaje, sino la necesidad de aplicarlas e identificar los efectos en la educación integral. Lo último debe ir más allá de aplicaciones generales y la descripción de las dimensiones de la Competencia Emocional, es decir, analizar las motivaciones, bienestar y cómo incide en la retroalimentación de conocimientos en el proceso de formación.

Es así que, los estudiantes de la carrera de Educación Básica de la Universidad Nacional de Loja se encuentran en edades promedio entre 19 y 23 años, quienes están inmersos a diferentes cambios emocionales y sociales, por lo cual es fundamental que desde la función docente se promueva el bienestar psicológico para brindarles experiencias significativas que ayuden a protegerlos de situaciones que alteren la capacidad de regularidad que causen presión y que influya en la alteración de cualquier aspecto emocional vinculadas con ansiedad, irritabilidad, frustración, arrebatos emocionales, dificultades para mantener relaciones interpersonales, bajo rendimiento académico, desmotivados y desinterés en la educación.

Atendiendo a lo anterior, el estudio mantiene como importancia bajo las competencias emocionales básicas en los docentes en formación, 
así como en las instituciones de Educación Superior, las cuales tienen como perspectiva mejorar las diferentes ofertas académicas desde los rediseños del programa de estudios de las carreras, por lo que se hace fundamental analizar los factores emocionales influyentes para que los futuros profesores durante el proceso formativo puedan progresar académicamente, también en competencias emocionales que permita desenvolverse exitosamente en la práctica profesional.

\section{MÉTODO}

Se realizó un estudio cuasi experimental exploratoria, teniendo como finalidad determinar las competencias emocionales básicas y la relación con el proceso de enseñanza y aprendizaje de los profesores en formación de la carrera de Educación Básica de la Universidad Nacional de Loja. La población estuvo conformada por los estudiantes de Educación Básica de la Universidad Nacional de Loja en Ecuador, donde se identificaron un total de 351 estudiantes matriculados. Sin embargo, debido a problemas de acceso se calculó una muestra, para ello se utilizó un tipo de muestreo probabilístico con población finita, quedando constituida por 99 estudiantes de educación básica de la Universidad objeto de estudio.

En la conceptualización de las variables se estableció como variable independiente percepción y comprensión de las emociones, expresión y clasificación de las emociones, manejo y regulación de emociones, conciencia emocional, regulación emocional, autonomía emocional, competencias sociales Competencias para la vida, el bienestar y por ultimo desempeño académico. Entre las variables dependientes se tuvo en cuenta: inteligencia emocional de los docentes y desarrollo emocional de los alumnos. Las variables analizadas fueron estudiadas, contrastadas estadísticamente al inicio y al final con base a la información base del grupo experimental y el grupo control.

El enfoque que predomino en el estudio fue mixto, (cualitativo y cuantitativo), realizándose tabulaciones de datos mediante la aplicación de cuestionarios a un sector de la población.

Se usó el cuestionario de inteligencia emocional para maestros (QIEP por sus siglas en portugués) el cual fue valorado en una escala de Likert de 1 a 6 , siendo 1 la opción que nunca realizan los docentes en el aula y 6 la acción que siempre realizan.

Se evaluaron tres dimensiones las cuales fueron la percepción y comprensión de las emociones, además la expresión y clasificación de las emociones, y finalmente el manejo y regulación de emociones.

Se aplicó el cuestionario de desarrollo emocional de adultos (CDE-A) el cual estuvo compuesto de cinco dimensiones que evalúan las competencias emocionales, a través de la conciencia emocional, la regulación emocional, autonomía emocional, competencias sociales y competencias para la vida y el bienestar valoradas en una escala de Likert, como valor mínimo $1=$ nunca y $6=$ siempre.

\section{RESULTADOS Y DISCUSIÓN}

A continuación se destacan los resultados arrojados en el cuestionario de inteligencia emocional para maestros según sus dimensiones.

\section{Cuestionario de inteligencia emocional para maestros}

Se muestra en la Tabla 1 que el 44,4\% (40) respondieron que a veces desarrollaban los conocimientos de las competencias emociónale en sus estudiantes, el 27,7\% (25) a menudo, siempre el $21,1 \%(19)$ y solamente el $6.6 \%$ (6) respondieron que nunca. 
Tabla 1. Maestros que poseen conocimientos necesarios para promover el desarrollo emocional en sus estudiantes.

\begin{tabular}{lcc}
\hline $\begin{array}{l}\text { Conocimientos de las competencias emocionales para } \\
\text { promover el desarrollo de los estudiantes }\end{array}$ & No & \% \\
\hline Nunca & 6 & 6.6 \\
A veces & 40 & 44.4 \\
A menudo & 25 & $27-7$ \\
Siempre & 19 & 21.1 \\
Total & $\mathbf{9 0}$ & $\mathbf{1 0 0}$ \\
\hline
\end{tabular}

En la Tabla 2 queda reflejado que un mayor porcentaje de maestros destacan a menudo empatía con sus estudiantes (40\%), el 35,5\% a veces muestra empatía y el otro $24,4 \%$ siempre, lo que indica una variabilidad en cuanto al manejo de las emociones.

Tabla 2. Empatía de los maestros con sus estudiantes.

\begin{tabular}{llc}
\hline Empatía de los maestros con sus estudiantes. & No & $\%$ \\
\hline Empatía & No & $\%$ \\
A veces & 32 & 35.5 \\
A menudo & 36 & $40-0$ \\
Siempre & 22 & $24-4$ \\
Total & $\mathbf{9 0}$ & $\mathbf{1 0 0}$ \\
\hline
\end{tabular}

Continuando con los resultados arrojados se puede apreciar en la Tabla 3 que le mayor porcentaje correspondió que los maestros a menudo y siempre aplican la estrategia de la escucha en el afrontamiento de situaciones.

Tabla 3. Maestros que permiten espacios para la escucha de las emociones de sus estudiantes.

\begin{tabular}{lcc}
\hline $\begin{array}{l}\text { Maestros que permiten espacios para la escucha de las } \\
\text { emociones de sus estudiantes }\end{array}$ & No & $\%$ \\
\hline A veces & 19 & 21.1 \\
A menudo & 40 & 44.4 \\
Siempre & 31 & 34.4 \\
Total & $\mathbf{9 0}$ & $\mathbf{1 0 0}$ \\
\hline
\end{tabular}

Con relación a la expresión de las emociones por parte de los maestros como se muestra en la Tabla 4 en las escalas a menudo y siempre con la expresión de los maestros de sus emociones en clases destacan con un $36,6 \%$ respectivamente, indicando además que el 8,8\% de los maestros nunca expresan bien sus emociones en clase. 
Tabla 4. Maestros que expresan bien sus emociones en clase.

\begin{tabular}{lcc}
\hline Maestros que expresan bien sus emociones en clase. & No & $\%$ \\
\hline Nunca & 8 & 8.8 \\
A veces & 16 & 17.7 \\
A menudo & 33 & 36.6 \\
Siempre & 33 & $36-6$ \\
Total & $\mathbf{9 0}$ & $\mathbf{1 0 0}$ \\
\hline
\end{tabular}

Continuando con los resultados arrojados se puede apreciar en la Tabla 3 que le mayor porcentaje correspondió que los maestros a menudo y siempre aplican la estrategia de la escucha en el afrontamiento de situaciones.

Tabla 5. Habilidad para describir los sentimientos de un estudiante a partir de su expresión facial.

\begin{tabular}{lcc}
\hline $\begin{array}{l}\text { Habilidad para describir los sentimientos de un } \\
\text { estudiante a partir de su expresión facial. }\end{array}$ & No & $\%$ \\
\hline A veces & 17 & 18.8 \\
A menudo & 45 & $50-0$ \\
Siempre & 28 & $31-1$ \\
Total & $\mathbf{9 0}$ & $\mathbf{1 0 0}$ \\
\hline
\end{tabular}

Con relación a que si el maestro expresa fácilmente el afecto hacia sus alumnos se obtuvo como resultado que la mayoría de los maestros con frecuencia son competente para el desarrollo de esta habilidad (70\%).

Con respecto a darse cuenta cuando un estudiante trata de ocultar sus verdaderos sentimientos solamente el $27,7 \%$ (25) desarrollan esta habilidad, se debe destacar que ninguno planteó que nunca la desarrollan.

La respuesta relacionada con la descripción de la mayoría de sus sentimientos la respuesta de los maestros fue que con frecuencia las cuentan 58,8\%.

La mayoría de los maestros plantearon que durante las clases tratan de moderar las emociones desagradables y reforzar las positivas, 75,5\% (68).
En relación al ítem referida a si creen que sus maneras de gestionar sus emociones pueden influir en el aula, el $85 \%$ de los maestros afirman que sin duda que su manera de gestionar sus emociones pueden influir en el aula y el $15 \%$ supone que si influye.

Resultados del cuestionario de desarrollo emocional de adultos

Al aplicar el cuestionario a los adultos, en la pregunta sobre si se siente una persona feliz, 50\% señaló la opción "a menudo".

Respecto a que si esta satisfecho con la manera de afrontar la vida y ser feliz, 68,8\% de los adultos tomaron como elección poca veces, $16,1 \%$ a veces y el $15,1 \%$ a menudo. 
Al aplicar el cuestionario a los adultos y analizar el ítem si esta satisfecho con la manera de afrontar la vida y ser feliz, se obtuvo que $71,1 \%$ lo identificaron a veces, $20,2 \%$ a menudo y solamente $8.0 \%$ siempre

Sobre si esta descontento consigo mismo, el $75,7 \%$ tomo la opción de rara vez, 19,1 \% a veces y el $6.0 \%$ refirió a menudo.

Al indagar sobre si los cambios lo asustan, $61,6 \%$ a veces, $22,1 \%$ a menudo y $16,1 \%$ siempre.

Por otra parte, la aplicación del cuestionario determinó que el 82,8\% a menudo al hablar con otras personas poco conocidas le resulta difícil, habla poco y siente nerviosismo y $17,1 \%$ siempre.
Del total de adultos, $12,1 \%$ siempre acepta y respeta que los otros piensen y actúen de forma diferente, el 62,6\% declara a veces y $25,2 \%$ nunca.

En la Tabla 6 se observa que el $35,3 \%$ de los adultos a menudo toman en cuenta los sentimientos de las demás personas, 25,2 \% nunca, 22\% siempre y el $17,1 \%$ a veces.

Tabla 6. Frecuencia del adulto que toman en cuenta los sentimientos de las demás personas.

\begin{tabular}{lcc}
\hline $\begin{array}{l}\text { Frecuencia del adulto que toman en cuenta los } \\
\text { sentimientos de las demás personas. }\end{array}$ & No & \% \\
\hline Nunca & 25 & 25.2 \\
A veces & 17 & 17.1 \\
A menudo & 35 & $35-3$ \\
Siempre & 22 & 22.2 \\
Total & $\mathbf{9 9}$ & $\mathbf{1 0 0}$ \\
\hline
\end{tabular}

\section{Discusión}

Como efecto de los vertiginosos avances de la ciencia, la tecnología y los complejos procesos globales de los últimos años, los sistemas educativos en el mundo y en la región latinoamericana, han venido replanteando no sólo los fines mismos de la educación y la escuela, sino las competencias que tanto los estudiantes (y sus familias), como los docentes, hoy deben desarrollar para afrontar la vida con sus cambios constantes e incertidumbre predominante en todos los órdenes; y en el caso particular del profesorado, además, para encontrar significado a su labor y poder desempeñarse con la eficacia que demanda la calidad educativa (Álvarez, 2019).
Estudiosos del tema Calderón et al., (2014) Plantean que la educación emocional busca que las personas tengan las herramientas necesarias para conocer, expresar y manejar sus propias emociones y las de las demás personas, de manera que estas no afecten sus vidas y que, por el contrario, promuevan el bienestar personal y social. Los datos arrojados en este estudio muestran escasez de estas herramientas tanto de los maestros como de los adultos.

Según Falus (2011), el profesorado debe tener conocimiento acerca de contenidos disciplinares, así como de las condiciones físicas, socio-afectivas y destrezas cognitivas de las niñas y los niños. Es decir, además de los contenidos didácticos, la persona profesional en docencia debe tener un 
conocimiento integral de la niña y el niño, no solo en la parte académica. Los resultados expuestos demuestran que la mayoría de los maestros no desarrollan los conocimientos acerca de las competencias emocionales.

Estudios como el de Hué, (2008) se articulan a los principios de la inteligencia emocional para proponer métodos para dar respuesta a la necesidad de formar a los docentes en el desarrollo de competencias emocionales que permita mejorar la competencia didáctica en las aulas, así aumentar el bienestar docente generando emociones y sentimientos positivos que remplacen a los negativos.

Pérez et al., (2010), analizaron la relación entre la competencia emocional y la social en estudiantes universitarios, en la cual se aplicó el Cuestionario de Desarrollo Emocional para Adultos (QDE-A) elaborado por el Grupo de investigación en Orientación Psicopedagógica (GROP) y la Escala de Habilidades Sociales (EHS) de Gismero, (2000). El estudio correlacional corrobora la relación entre competencia emocional y competencia social una persona competente a nivel emocional; probablemente lo es a nivel social, ya que las habilidades sociales aparecen fuertemente relacionadas con la competencia emocional que se relaciona con niveles óptimos de desempeño en ámbito personal, educativo, profesional y en el afrontamiento exitosos de los retos en la vida diaria.

Favorecer los espacios áulicos y el contexto educativo en general, a través de un programa basado en competencias emocionales admite que el aprendizaje sea significativo, los estudiantes como docentes en formación adquieren competencias y habilidades emocionales para identificar, entender y gestionar las emociones que guie los procedimientos formativos, curriculares de cada contexto y sistema educativo, siendo empáticos, reflexivos, mediadores en el conflicto, responsable de las decisiones y autoconocimiento de sus emociones.

$\mathrm{Si}$ ciertamente los maestros requieren de una formación académica y profesional que les garantice su buen hacer docente, resulta también imprescindible que tengan una correcta preparación en todo aquello que gire alrededor de los afectos y de las emociones, tanto suyas como de los demás. Aquí aparecen junto a las competencias académicas y profesionales, las competencias emocionales Signes et al., (2014). Los resultados de esta investigación reafirma este planteamiento.

Es por ello que, se considera la importancia de las competencias emocionales básicas en los docentes en formación, así como las instituciones de Educación Superior tienen la perspectiva de mejorar las diferentes ofertas académicas desde los rediseños del programa de estudios de las carreras, se hace fundamental analizar los factores emocionales influyentes para que los futuros profesores durante el proceso formativo puedan progresar académicamente, también en competencias emocionales que permitirá desenvolverse exitosamente en la práctica profesional.

\section{CONCLUSIONES}

Se concluye la pertinencia de brindar al personal docente un modelo de aprendizaje que comprende la motivación, las estrategias de aprendizaje e inteligencia emocional, aplicarlo y analizar las competencias emocionales de los estudiantes en formación de la Carrera de Educación Básica antes y después de la aplicación del modelo en un contexto de pandemia. 
REFERENCIAS BIBLIOGRÁFICAS

Álvarez, E. (2019). La competencia emocional como reto en la formación y actualización docente. Reflexiones a partir de un estudio de casos en educación básica en México. ALAS-Asociación Latinoamericana de Sociología, 10(17)

Argudin, Y. (2005). Educación basada en competencias. Trillas

Bisquerra, R., y Pérez, N. (2007). Las competencias emocionales. Educación XXI, 61-82

Cáceres, I. (2020). Educación en el escenario actual de pandemia. Revista internacional de filosofía y teoría social, 25(5), 11-12

Calderón, M.; González, G., Salazar, P., y Washburn, S. (2014). EL PAPEL DOCENTE ANTE LAS EMOCIONES DE NIÑAS Y NIÑOS DE TERCER GRADO. Revista Electrónica "Actualidades Investigativas en Educación, 14(1), $1-23$

Camargo, C. P., Tempski, P. Z., Busnardo, F. F., Martins, M. A., y Gemperli, R. (2020). Online learning and COVID-19: a meta-synthesis analysis. Clinics. Clinics (Sao Paulo, Brazil), 75, 22-86. https://doi.org/https://doi.org/10.6061/ clinics/2020/e2286

García, E. (2017). Formación del profesorado en educación emocional: diseño, aplicación $y$ evaluación. Universidad de Barcelona
Gismero, E. (2000). EHS: Escala de Habilidades Sociales. TEA

Hué, C. (2008). Bienestar docente y pensamiento emocional. Wolters Kluwer

Pegalajar, M., y López, L. (2015). Competencias emocionales en el proceso de formación del docente de educación infantil. Revista Iberoamericana sobre Calidad, Eficacia y Cambio en Educación, 13(3), 95-105

Pérez, N., Filella, G., y Soldevilla, A. (2010). Competencia emocional y habilidades sociales en estudiantes universitarios. Revista Electrónica de Motivación y Emoción, XIII(34)

Sánchez, L. (2019). La formació inicial docente universitaria chilena: estudiando el ámbito emocional. Praxis \& Saber, 10(24), 217-242

Signes, M. T., Amado, L., Acereda, A., y Lopez, A. (2014). Desarrollo de las competencias emocionales del maestro: eje vertebrador del proceso de enseñanza aprendizaje. Revista Internacional de Educación y Aprendizaje, 2(2), 27-36

Torrijos, P. (2016). Desarrollo y evaluación de competencias emocionales para profesores mediante una intervención por programas. Universidad de Salamanca

UNESCO. (2020). Respuesta del ámbito educativo de la UNESCO al COVID-19 